\title{
Applying First-Order Perturbation Theory of Quantum Mechanics to Predict and Build a Postprandial Plasma Glucose Waveform (GH-Method: Math- Physical Medicine)
}

\author{
Gerald C Hsu* \\ Eclaire MD Foundation, USA \\ ${ }^{*}$ Corresponding author: Gerald C Hsu, Eclaire MD Foundation, USA; Email: g.hsu@eclairemd.com
}

Received: April 06, 2020; Accepted: April 14, 2020; Published: April 21, 2020

\section{Introduction}

In this paper, the author presents his techniques of applying firstorder perturbation theory of quantum mechanics to predict and build a Postprandial Plasma Glucose (PPG) waveform based on the "perturbation factor" of carbs/sugar intake amount. This is a part of his GH-Method: math-physical medicine research methodology.

\section{Methods}

Initially, he applied segmentation pattern analysis to analyze his 1,825 meals with 23,725 PPG Sensor data collected during a period of 5/5/201812/13/2019. Initially, his two segments were based on both "first factor" of meal's carbs/sugar intake amounts and "second factor" of post-meal walking steps. His low-carb meals occupy about $2 / 3$ of the total meals $(1,209$ meals with 8.5 grams per meal) and high-carb meals occupy about $1 / 3$ of the total meals ( 615 meals with 27.1 grams per meal). A standard waveform (curve) contains 13 data points for each PPG curve and one input data for each 15 -minute time segment. His post- meal walking steps are comparable (4,238 vs. 4,282 steps). Therefore, he decided to focus on the first factor of carbs/sugar intake amount only.

Next, he applied the first-order perturbation theory of quantum mechanics to continue and extend his glucose prediction research work. The perturbation equation is expressed in the following:

$$
\mathrm{A}=\sim\left(\mathrm{A} 0+\varepsilon^{*} \mathrm{~A} 1\right)
$$

Where A0 would be the known solution to a simpler but solvable initial problem and A1 represents the first-order term which may be found interactively by some systematic procedure. For small $\varepsilon$ (epsilon), this higher-order term in the series becomes successively smaller and derives to an approximate solution.

Since the second factor of post-meal walking steps are almost equal ( 4,238 vs. 4,282 steps) between the low-carb case and high-carb case, he will only focus on the first factor of carbs/sugar intake amount. The author conducted the two following perturbation analysis cases:

(1) Using a combination of weighted carbs/sugar amount, 14.6 grams, which is equal to $\left(1 / 3{ }^{*}\right.$ high-carbs $+2 / 3 *$ low-carbs $)$.
(2) Using an average carbs/sugar amount, 17.8 grams, which is equal to $1 / 2 *$ (high-carbs + low-carbs)

He will then be able to construct two new separate PPG waveforms (curves) between 0 -minute throughout 180 -minutes by applying the perturbation theory.

Finally, he used his collected data to calculate and construct a waveform with a gram of carb following very closely to the perturbed waveform with 17.8 grams of carb.

\section{Results}

Figures 1 and 2 display both the data table and waveforms chart of low-carb pattern vs. high-carb pattern. Although their opening glucoses at 0 -minute $(129 \mathrm{mg} / \mathrm{dL}$ vs. $131 \mathrm{mg} / \mathrm{dL})$ and PPG curve shapes

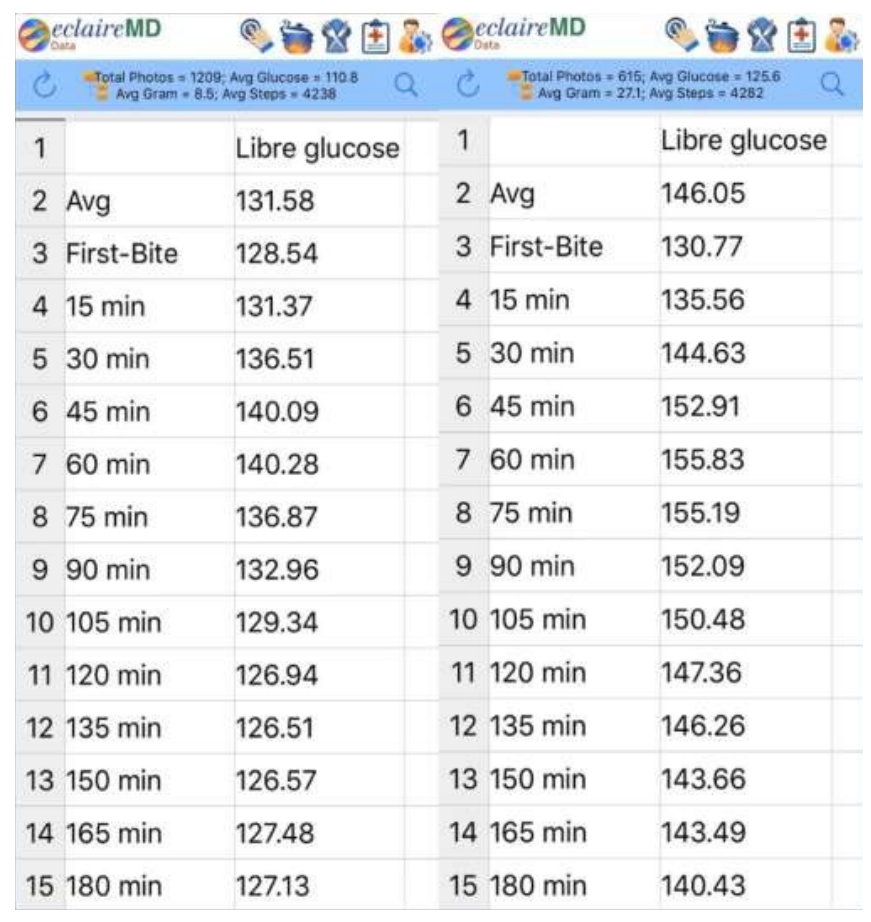

Figure 1: Data table of low-carbs and high-carbs PPG values. 


\section{PPG Waveform Differences (Segmentation Analysis)}

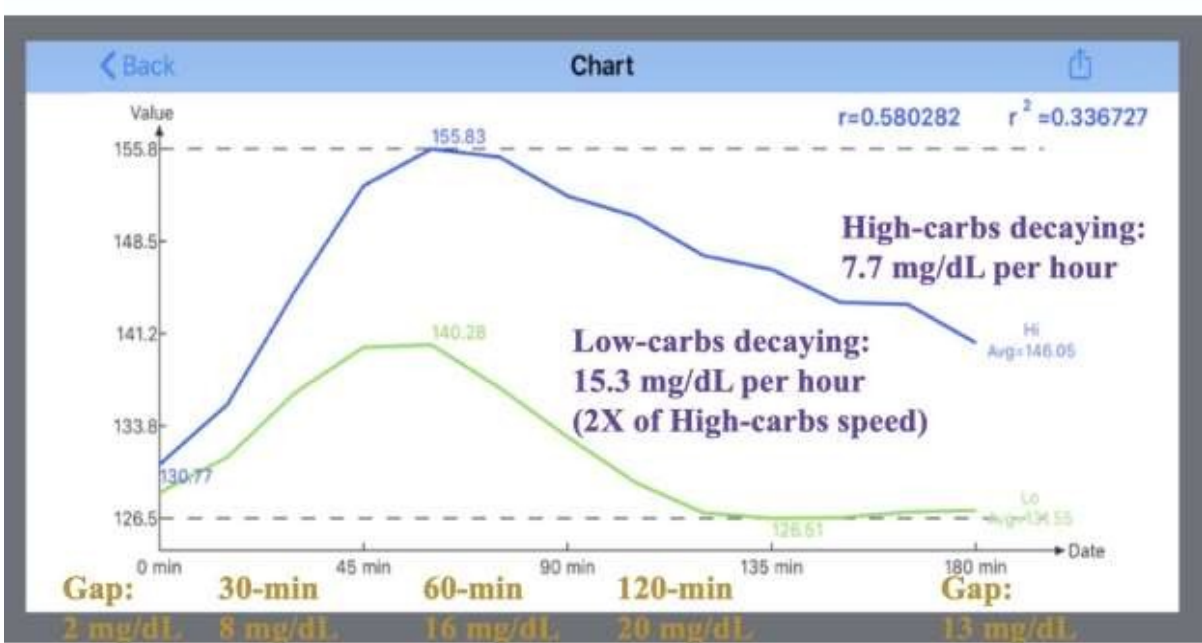

eclaire MD

Figure 2: Graphic chart of low-carbs and high-carbs waveforms.

are quite similar (two "mountain" shapes with 58\% correlation), their peak glucoses $(140 \mathrm{mg} / \mathrm{dL}$ vs. $156 \mathrm{mg} / \mathrm{dL})$ and closing glucoses at $180-$ minutes $(127 \mathrm{mg} / \mathrm{dL}$ vs. $140 \mathrm{mg} / \mathrm{dL})$ have different results. These differences have resulted from varying glucose decaying speeds after 60-minutes, which have deeper biomedical meanings, and are extremely critical to a patient's risk probabilities of having diabetes complications. The significance of these differences from a segmentation analysis has already been discussed in his previous publications and presentations.

Figures 3 and 4 illustrate both data table and two additional "perturbed waveforms" between low-carbs and high-carbs. It should be noted that the weighted combination PPG curve (14.6g) almost completely matches with the original PPG curve generated with real data. However, the average carb PPG curve (17.8g) is a newly generated

\begin{tabular}{|c|c|c|c|c|c|c|c|}
\hline Cancel & \multicolumn{2}{|c|}{ Self Defined Data } & OK & Cancel & Self Defined Data & ined Data & OK \\
\hline Date & (D) Low & High & $17.8 \mathrm{~g}$ & Date & $\infty$ Low & (2) High & (2) $14.6 \mathrm{~g}$ \\
\hline $0 \mathrm{~min}$ & 128.54 & 130.77 & 129.66 & $0 \mathrm{~min}$ & 128.54 & 130.77 & 129.23 \\
\hline $15 \min$ & 131.37 & 135.56 & 133.47 & $15 \mathrm{~min}$ & 131.37 & 135.56 & 132.70 \\
\hline $30 \mathrm{~min}$ & 136.51 & 144.63 & 140.57 & $30 \mathrm{~min}$ & 136.51 & 144.63 & 139.14 \\
\hline $45 \mathrm{~min}$ & 140.09 & 152.91 & 146.50 & $45 \mathrm{~min}$ & 140.09 & 152.91 & 144.31 \\
\hline $60 \mathrm{~min}$ & 140.28 & 155.83 & 148.06 & $60 \mathrm{~min}$ & 140.28 & 155.83 & 145.44 \\
\hline $75 \mathrm{~min}$ & 136.87 & 155.19 & 146.03 & $75 \mathrm{~min}$ & 136.87 & 155.19 & 142.87 \\
\hline 90 min & 132.96 & 152.09 & 142.53 & $90 \mathrm{~min}$ & 132.96 & 152.09 & 139.29 \\
\hline $105 \min$ & 129.34 & 150.48 & 139.91 & 105 min & 129.34 & 150.48 & 136.20 \\
\hline $120 \mathrm{~min}$ & 126.94 & 147.36 & 137.15 & $120 \mathrm{~min}$ & 126.94 & 147.36 & 133.66 \\
\hline $135 \mathrm{~min}$ & 126.51 & 146.26 & 136.39 & $135 \mathrm{~min}$ & 126.51 & 146.26 & 132.99 \\
\hline $150 \mathrm{~min}$ & 126.57 & 143.66 & 135.12 & $150 \mathrm{~min}$ & 126.57 & 143.66 & 132.11 \\
\hline $165 \mathrm{~min}$ & 127.48 & 143.49 & 135.49 & $165 \mathrm{~min}$ & 127.48 & 143.49 & 132.63 \\
\hline $180 \mathrm{~min}$ & 127.13 & 140.43 & 133.78 & $180 \mathrm{~min}$ & 127.13 & 140.43 & 131.36 \\
\hline
\end{tabular}

Figure 3: Data table of low-carbs, high-carbs, and two perturbed PPG values. 


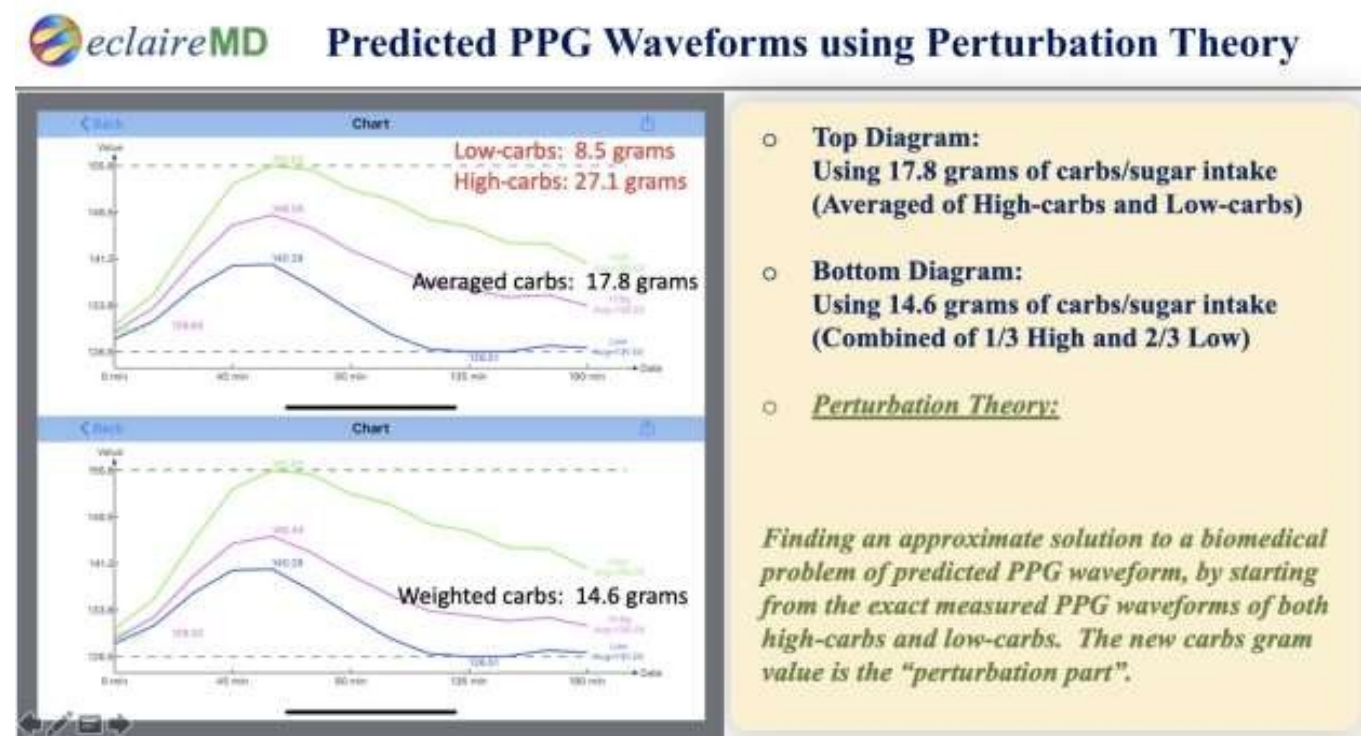

Figure 4: Graphic chart of low-carbs, high-carbs, and two perturbed PPG waveforms.

waveform by using the perturbed factor, carbs amount, which is similar to those two original curves, low-carbs and high-carbs, but are not the same if you examine them closely. Through application of perturbation theory concept of quantum mechanics, the author could generate a predicted PPG waveform entirely based on the selected "perturbation factor" of 17.8 grams of carbs/sugar intake amount. Of course, this perturbed waveform is only an approximated curve based on the first perturbation factor, carbs/sugar intake amount.
For clarity of waveform comparison, Figure 5 further demonstrates these two newly generated perturbed waveforms by using two slightly different perturbed carb values.

In figures 6 and 7, the author selected 84 meals with an averaged carb amount of $18 \mathrm{~g}$ and constructed a new waveform between $0-180$ minutes. This measured PPG waveform with $18 \mathrm{~g}$ and $138.21 \mathrm{mg} / \mathrm{dL}$ is compared against the perturbed PPG waveform with $17.8 \mathrm{~g}$ and

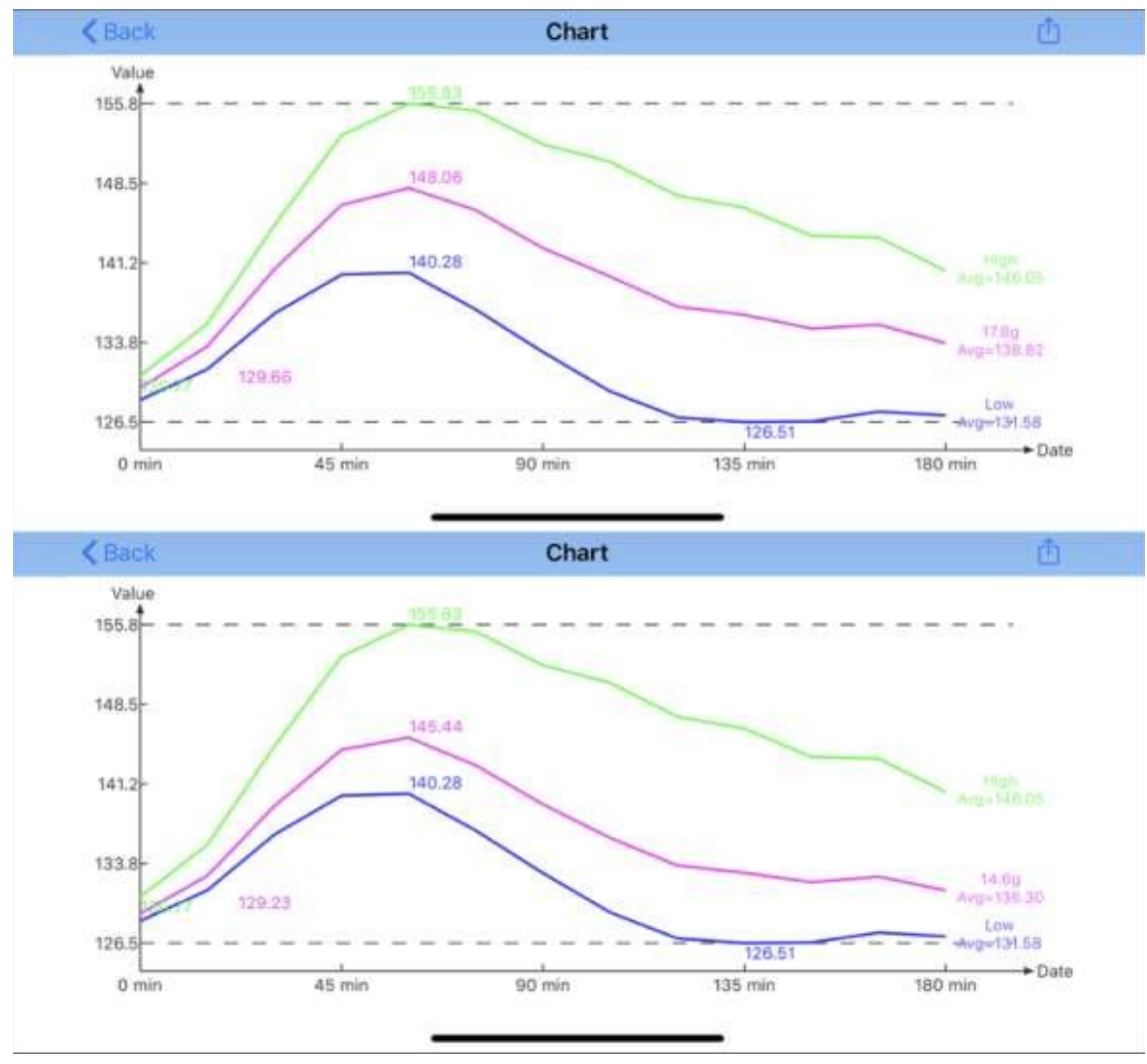

Figure 5: Waveforms comparison. 


\begin{tabular}{|c|c|c|c|}
\hline \multicolumn{2}{|c|}{$Q_{\text {Data }}^{\text {eclaireMD }}$} & \multicolumn{2}{|c|}{ ( } \\
\hline Cancel & \multicolumn{2}{|c|}{ Self Defined Data } & OK \\
\hline Date & $17.8 \mathrm{~g}$ & $18 \mathrm{~g}$ & $18-17$ \\
\hline $0 \mathrm{~min}$ & 129.66 & 128.67 & -0.99 \\
\hline $15 \mathrm{~min}$ & 133.47 & 133.73 & 0.26 \\
\hline $30 \mathrm{~min}$ & 140.57 & 141.60 & 1.03 \\
\hline $45 \mathrm{~min}$ & 146.50 & 148.19 & 1.69 \\
\hline $60 \mathrm{~min}$ & 148.06 & 149.28 & 1.22 \\
\hline $75 \mathrm{~min}$ & 146.03 & 147.58 & 1.55 \\
\hline $90 \mathrm{~min}$ & 142.53 & 142.02 & -0.51 \\
\hline $105 \mathrm{~min}$ & 139.91 & 140.46 & 0.55 \\
\hline $120 \mathrm{~min}$ & 137.15 & 136.28 & -0.87 \\
\hline $135 \mathrm{~min}$ & 136.39 & 135.12 & -1.27 \\
\hline $150 \mathrm{~min}$ & 135.12 & 130.21 & -4.91 \\
\hline $165 \mathrm{~min}$ & 135.49 & 131.87 & -3.62 \\
\hline $180 \mathrm{~min}$ & 133.78 & 130.49 & -3.32 \\
\hline
\end{tabular}

Figure 6: Verification data comparison between measured PPG @ 18g and perturbed PPG @ $17.8 \mathrm{~g}$.
$138.81 \mathrm{mg} / \mathrm{dL}$ to achieve a combined (adjusted) prediction accuracy of $98.4 \%$.

The deviation amount of PPG is $0.5 \%$ and the deviation amount of carbs gram is $1.1 \%$ (in an opposite direction). Therefore, the combined deviation is $1.6 \%$, which yields an accuracy rate of $98.6 \%$.

\section{Conclusion}

Glucose variance is an extremely complex biochemical and biophysical phenomenon. After a diabetes patient measures and establishes two separate initial waveforms with one low-carb meal and another high-carb meal separately, we can then collect the patient's PPG data and draw two separate PPG waveforms accordingly. As a result, we can predict the glucose behavior by using the perturbation theory of quantum mechanics to obtain an approximated PPG waveform according to this selected carbs/sugar intake. Of course, the same method can also be applied using the second per nation factor, post-meal waking steps. In this way, a patient will have the ability to predict his/her own PPG behavior before consuming a meal or initiate post-meal exercise.

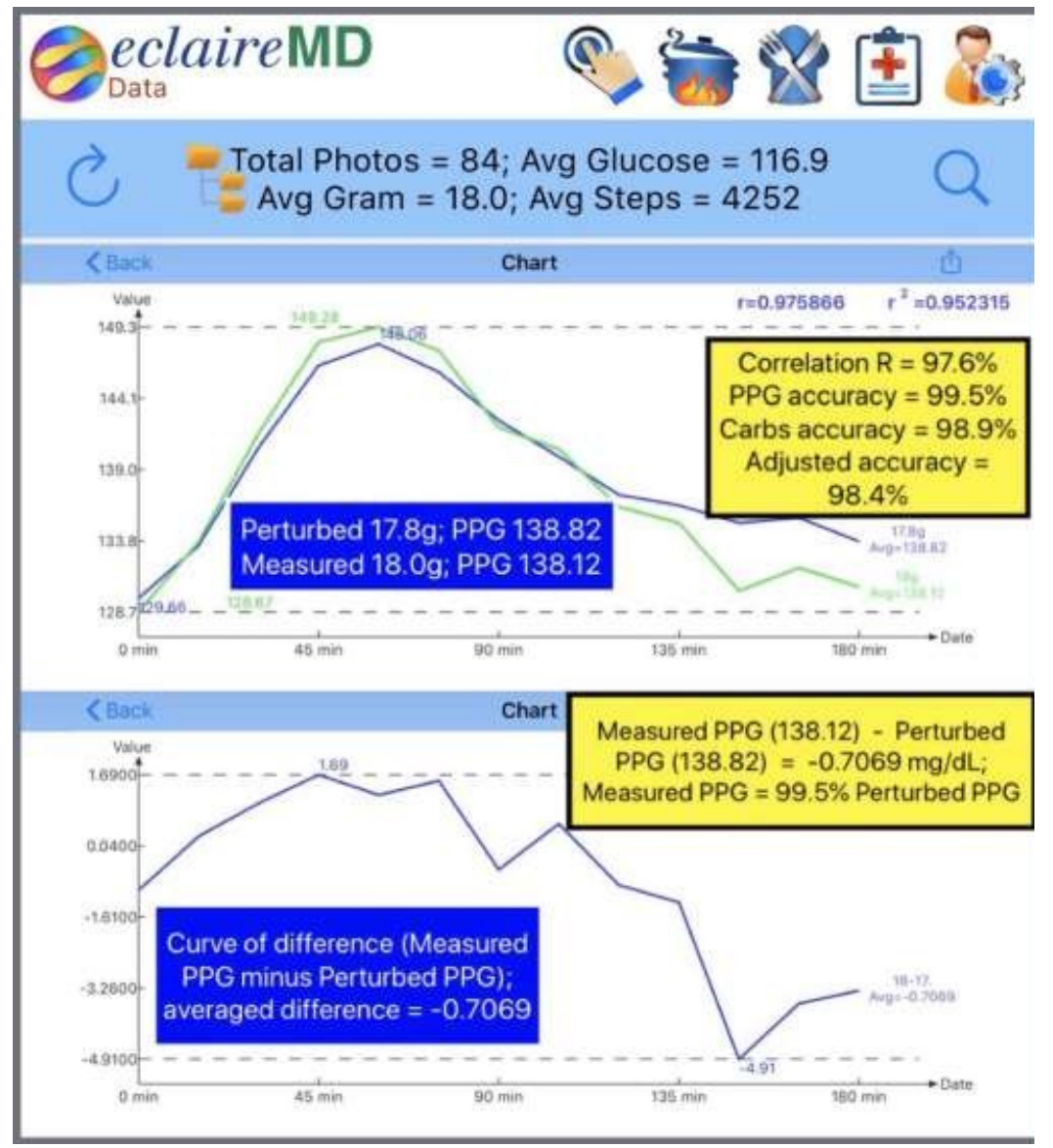

Figure 7: Verification waveform comparison between measured PPG and perturbed PPG plus a 98.4\% combined accuracy using perturbation prediction theory.

\section{Citation:}

Gerald C Hsu (2020) Applying First-Order Perturbation Theory of Quantum Mechanics to Predict and Build a Postprandial Plasma Glucose Waveform (GH-Method: Math- Physical Medicine). Internal Med Res Open J Volume 5(2): 1-4. 